\title{
Bilateral superior keratoconus: two case reports
}

\begin{abstract}
Purpose To present two cases of the rare entity of bilateral superior keratoconus and to review the literature regarding presentation and clinical findings. Methods Case report based on chart review. Results Two patients presented to our corneal service with clinical and topographical features of superior keratoconus.

Conclusion The unusual clinical and topographical findings of superior keratoconus are presented and treatment options are considered.

Eye (2014) 28, 1254-1257; doi:10.1038/eye.2014.146;

published online 4 July 2014
\end{abstract}

\section{Introduction}

Keratoconus is a usually bilateral, often asymmetrical, non-inflammatory corneal ectasia that is progressive into early middle age. This ectasia causes corneal steepening with irregular asymmetrical astigmatism most typically seen in the infero-temporal paracentral zone. We report two rare cases of bilateral superior keratoconus accompanied by refractive maps acquired on the Pentacam HR anterior segment tomographer (Oculus GmBH, Wetzlar, Germany) at our unit.

\section{Case reports}

\section{Case 1}

A 26-year-old black African patient was referred to our corneal clinic with progressive loss of vision in her right eye and pre-existing poor vision in her left. She had previously been treated medically elsewhere for corneal hydrops in her left eye 3 years prior. A rigid gaspermeable contact lens was fitted previously OU but she had refused to wear them due to extreme discomfort upon insertion. Spectacles were provided without significant visual improvement. At that time uncorrected visual acuity was 6/9-3 OD and 3/60 OS. The patient was then lost to follow-up for 2 years.

With worsened keratometry on her right eye on re-presentation to her general ophthalmologist, she was referred to our department for further evaluation and consideration for corneal collagen cross-linking.

Questioning revealed only occasional eye rubbing and an intermittent itchy nose. She demonstrated an eye-rubbing technique over her upper lids using the knuckle of her index finger of her right hand. She also described performing a counter clockwise digital 'twisting' of her right eye to read subscript on the television. She was using treatment for asthma but otherwise had an unremarkable medical history.

It was not known whether she had any family history of keratoconus and all family members were reported to have good uncorrected vision.

Best spectacle-corrected visual acuity on presentation to our department had deteriorated to 6/18-3 OD and counting fingers at $3 \mathrm{~m}$ OS.

Marked scissoring was noted on retinoscopy. Bilateral inverse Munson's signs were noticed on upgaze. The lids were otherwise normal.

Slit-lamp biomicroscopy revealed superior corneal thinning OU. There were superior Vogt striae, deep stromal scars, and underlying vertical Descemet's membrane breaks OS. There were faint Fleischer rings OU noticed only on cobalt blue illumination. The central corneas in both eyes were clear with no inflammation, scarring, or vascularisation present. There was no evidence of current or previous allergic keratoconjunctivitis. The lenses were clear with no posterior segment abnormalities OU.

Pentacam HR anterior segment tomography demonstrated significant superior thinning $\mathrm{OU}$ with superior steepening OD. The sagittal curvature map OS was distorted due to previous superior hydrops and the resultant corneal scarring (Figures 1a and b). 

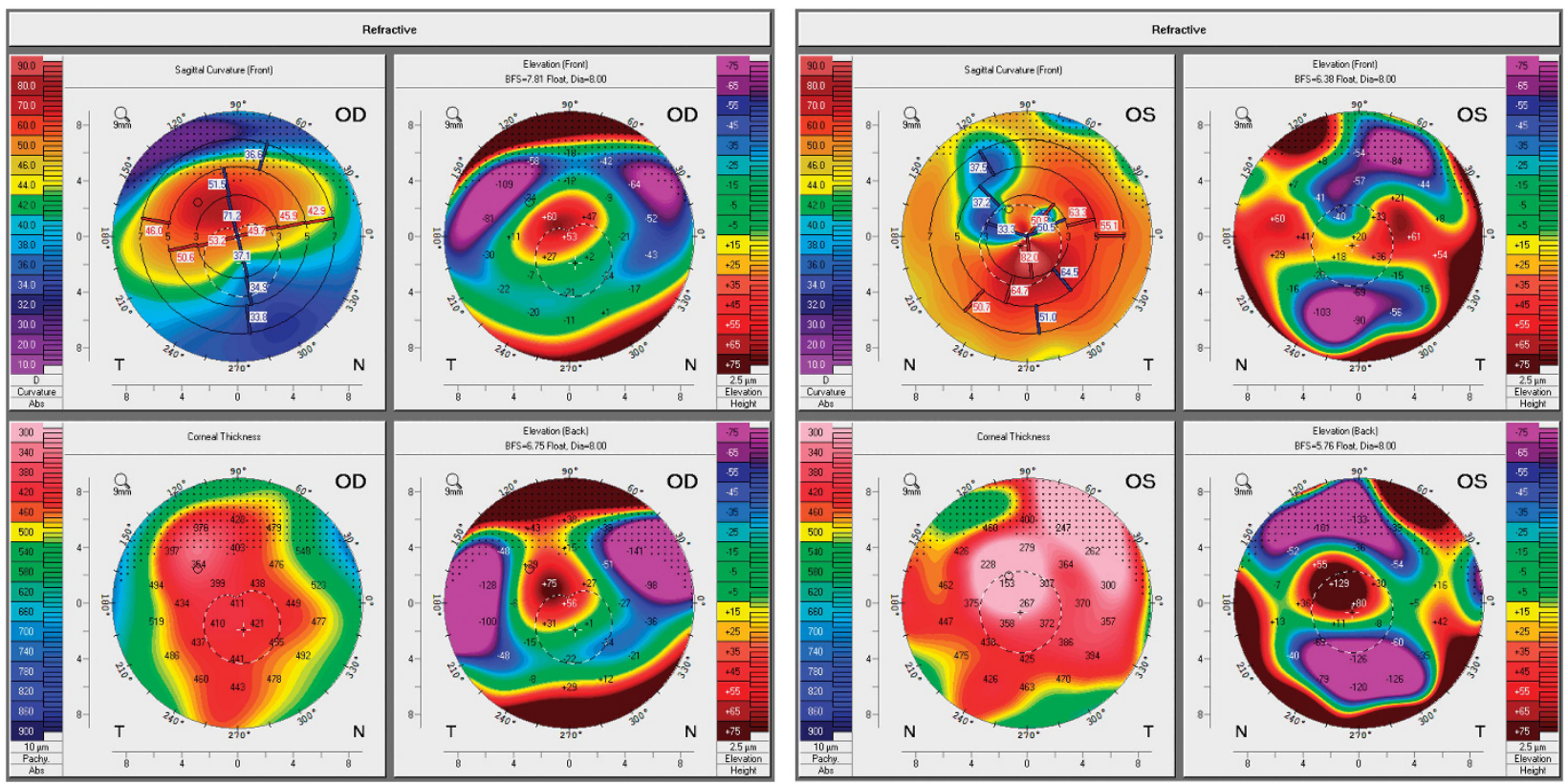

Figure 1 Pentacam-refractive maps of the right and left eyes of Case 1.

A thinnest point of $395 \mu \mathrm{m}$ was $1.22 \mathrm{~mm}$ superior and $1.43 \mathrm{~mm}$ temporal to the apex at the $135^{\circ}$ meridian OD. A thinnest point of $144 \mu \mathrm{m}$ was $0.98 \mathrm{~mm}$ superior and $0.65 \mathrm{~mm}$ nasal to the apex at the $120^{\circ}$ meridian OS. Both of these points were $>2 \mathrm{~mm}$ superior to the pupil centre. No superior marginal thinning was evident. There was inferior displacement of the pupil centre relative to the corneal apex OU.

The points of maximal posterior elevation in relation to the best-fit sphere of $74 \mu \mathrm{m}$ OD and $129 \mu \mathrm{m}$ OS were in direct relation to the location of the thinnest points OU.

Arrangements were made for corneal collagen crosslinking OD to be followed by a trial of rigid gaspermeable contact lens fitting after keratometric stability. A penetrating keratoplasty is planned OS as deep anterior lamellar keratoplasty is not possible due to Descemet's membrane breaks and the patient's complete intolerance to any form of contact lens or spectacle correction in that eye.

\section{Case 2}

An 18-year-old female scholar of mixed ethnicity also presented with a history of gradual loss of vision in both eyes. This loss of vision had first been noticed the previous year during a pregnancy. She denied using spectacles or contact lenses and had not reported previously having had poor vision while reading the blackboard at school.
Her medical history was otherwise unremarkable and she also gave no family history of keratoconus.

Her best spectacle-corrected visual acuity was 6/15 in both eyes. Both eyes were itchy although no regular topical or systemic medication had previously been used. Again, a right-handed index finger knuckle rub on the upper lid OU was demonstrated.

There was no obvious abnormality on external examination except a mild inverse Munson's sign and distinct scissoring on retinoscopy OU. On slit-lamp biomicroscopy, there was no active keratoconjunctivitis and both corneas were clear except for bilateral Fleischer rings noticed on cobalt blue examination only.

Oculus Pentacam HR images (Figures 2a and b) are included. Superior steepening and irregular asymmetric astigmatism are noted in both eyes.

The thinnest points were $459 \mu \mathrm{m}$ OD at the $120^{\circ}$ meridian and $492 \mu \mathrm{m}$ OS at $85^{\circ}$, both $>1 \mathrm{~mm}$ superior to the pupil centre but also marginally superior to the corneal apex, possibly implying slight downward gaze to obtain the clearest fixation on the Pentacam HR fixation target through the centre of the superiorly displaced cone. The points of maximal elevation on the posterior elevation maps were $68 \mu \mathrm{m}$ OD and $46 \mu \mathrm{m}$ OS anterior to the best-fit sphere and both located superior to the corneal apex.

As in Case 1, there is a direct correlation between the points of maximal posterior elevation and the 

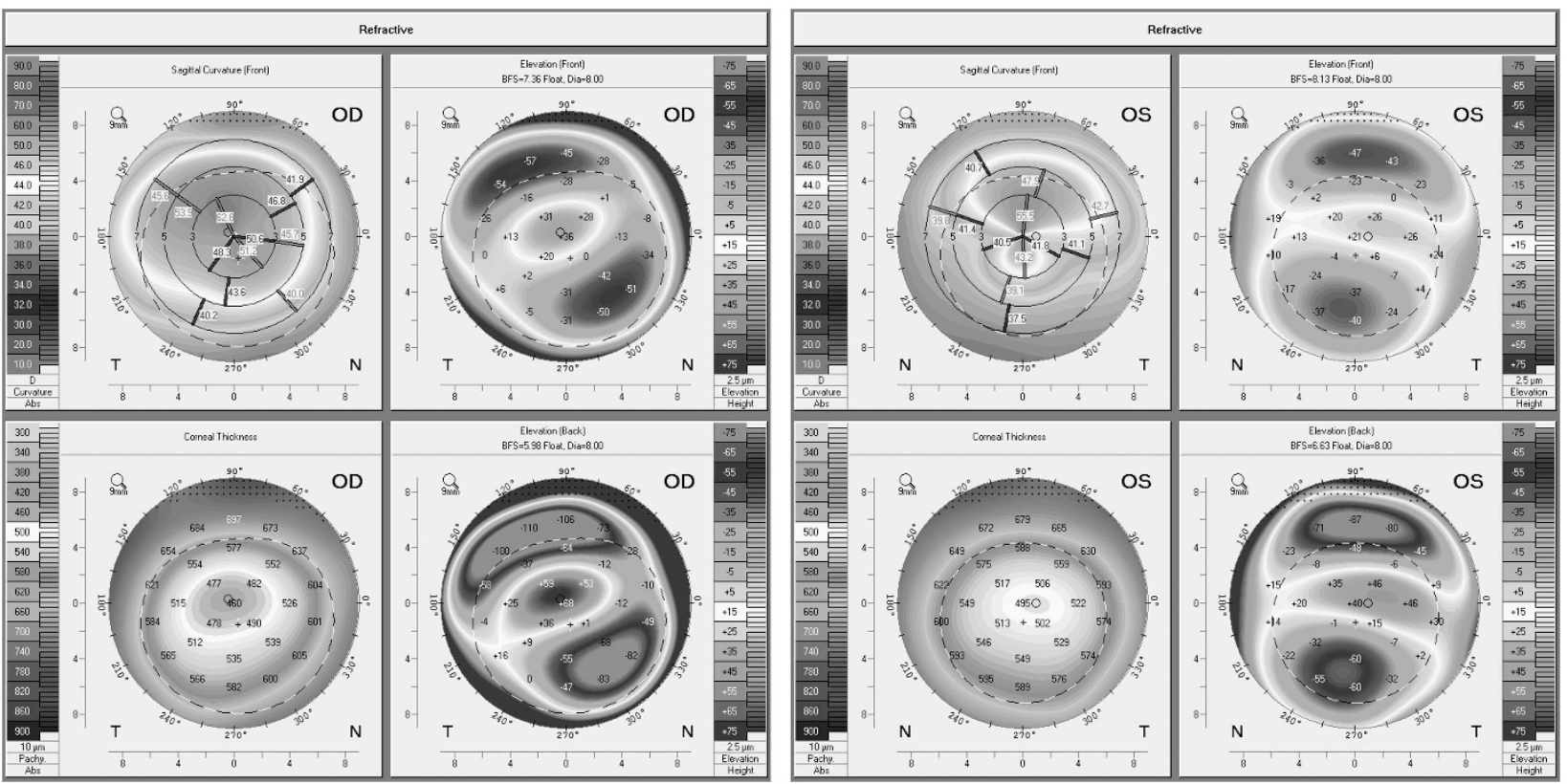

Figure 2 Pentacam-refractive maps of the right and left eyes of Case 2.

thinnest corneal points in both eyes. This patient underwent a trial of rigid gas-permeable contact lenses fitting.

\section{Discussion}

Keratoconus is a progressive, predominantly bilateral non-inflammatory ectatic disorder of the cornea. The protruding corneal apex and the cornea's thinnest points are paracentral and almost always inferior to the visual axis. This condition may result in poor uncorrected vision on the basis of irregular and/or asymmetric astigmatism. True superior keratoconus is extremely rare, comprising $<1$ per cent of cases of keratoconus. ${ }^{1}$ Only five previous isolated cases have been described in the literature. ${ }^{1-4}$ Other conditions causing superior corneal thinning and protrusion were excluded on biomicroscopy by the absence of lipid deposition or vascularisation. Also, the presence of a cone and the absence of a peripheral band of thinning eliminated superior pellucid marginal degeneration, the most likely alternative.

These patients presented with bilateral asymmetrical superior keratoconus. The first case had evidence of previous hydrops in the left eye. This is to our knowledge the most severe case documented to date.

Prisant et $a l^{2}$ and Eiferman et $a l^{3}$ reported similar cases of bilateral asymmetrical superior keratoconus; however, both cases had one subclinical eye and these were detected only using computerised video photokeratography. Otherwise, the most convincing clinical case of isolated superior keratoconus is that reported by Tananuvat et al, ${ }^{4}$ also markedly asymmetrical but the thinning was central rather than superior. In our case the apex of the cone and the thinnest points are patently superior and not central (as in that reported by Tananuvat et $a l^{4}$ ) or inferior (as in Chiang et $a l^{5}$ ) as well as being present in both eyes.

In a textbook by Barbara et al, the diagnosis of true superior keratoconus has been doubtful in several cases. ${ }^{6}$ The normalisation of previous superior corneal steepening after lid surgery makes corneal warpage by the lid position more probable than keratoconus in the case published by Kim et al. ${ }^{7}$

Other authors have described cases of combined pellucid marginal degeneration and superior keratoconus. ${ }^{5,8}$ In this paper the clinical signs and sequential Pentacam images conclusively demonstrate the presence of isolated bilateral superior keratoconus in these patients.

Mechanical trauma in genetically susceptible patients has been implicated in the pathogenesis of keratoconus. ${ }^{6}$ We requested demonstrations of the eye rubbing to determine if an atypical 'lower eyelid technique' may explain the superior location of the cone in this uncommon phenotype. The conscious demonstrated technique may, however, differ markedly from an habitual one. ${ }^{9}$ It is also possible that eye rubbing is not a major contributor in our patients consistent with the absence of other signs of ocular allergy. 


\section{Summary}

What was known before

- Bilateral superior keratoconus is a rare entity comprising $<1 \%$ of all cases of keratoconus.

- Although there have been a handful of published cases of superior keratoconus, many of these have been disputed, have been associated with other conditions, or have been unilateral.

\section{What this study adds}

- This is the first and possibly most severe case series of bilateral superior keratoconus in the literature to our knowledge.

- Each case is supported by oculus Pentacam HR images supporting the diagnoses.

- An atypical habitual eye-rubbing technique did not seem to be responsible for the configuration of ectasia in these cases.

\section{Conflict of interest}

The authors declare no conflict of interest.

\section{References}

1 Weed KH, McGhee CN, MacEwen CJ. Atypical unilateral superior keratoconus in young males. Cont Lens Anterior Eye 2005; 28: 177-179.

2 Prisant O, Legeais JM, Renard G. Superior keratoconus. Cornea 1997; 6: 693-694.

3 Eiferman RA, Lane L, Law M, Fields Y. Superior keratoconus. Refract Corneal Surg 1993; 9: 394-395.

4 Tananuvat N, Leeungurasatien P, Wiriyaluppa C. Superior keratoconus with hydrops. Int Ophthalmol 2009; 29: 419-421.

5 Chiang C-C, Lin J-M, Tsai Y-Y. Superior keratoconus with inferior paracentral corneal thinning and inferior peripheral pellucid marginal degeneration. Eye 2007; 21: 266-268.

6 Barbara A. Textbook on Keratoconus: New Insights. Jaypeed Digital: New Delhi, 2012.

7 Kim T, khosla-Gupta B, Debacker C. Blepharoptosis-induced superior keratoconus. Am J Ophthalmol 2000; 30: 232-234.

8 Cameron JA, Mahmood MA. Superior corneal thinning with pellucid marginal degeneration. Am J Ophthalmol 1990; 109: $486-487$.

9 McMonnies CW, Boneham GC. Keratoconus, allergy, itch, eye-rubbing and hand-dominance. Clin Exp Optom 2003; 86(6): 376-384. 